\section{Air pressure changes in the creation and bursting of the type-1 big bubble in deep anterior lamellar keratoplasty: an ex vivo study}

SL AlTaan, I Mohammed, DG Said and HS Dua
Larry A Donoso Laboratory for Eye Research, Academic Section of Ophthalmology, Division of Clinical Neuroscience, University of Nottingham, Nottingham, UK

Correspondence: HS Dua, Department of Ophthalmology, B Floor, Eye ENT Centre, Queens Medical Centre, Derby Road, Nottingham, NG7 2UH, UK

Tel: +44 (0)115924 9924; Fax: +44 (0)1159709963. E-mail: Harminder.dua@ nottingham.ac.uk

Received: 15 January 2017 Accepted in revised form: 4 May 2017 Published online: 30 June 2017
Abstract

Purpose To measure the pressure and volume of air required to create a big bubble (BB) in simulated deep anterior lamellar keratoplasty (DALK) in donor eyes and ascertain the bursting pressure of the BB. Patients and methods Twenty-two human sclera-corneal discs were used. Air was injected into the corneal stroma to create a BB and the pressure measured by means of a pressure converter attached to the system via a side port. A special clamp was designed to prevent air leak from the periphery of the discs. The pressure at which air emerged in the corneal tissue; the bursting pressure measured after advancing the needle into the bubble cavity and injecting more air; the volume of air required to create $\mathrm{A} B \mathrm{~B}$ and the volume of the $B B$ were ascertained. Results Type-1 BB were achieved in 19 and type-2 BB in 3 eyes. The maximum pressure reached to create a BB was $96.25+/-$ $21.61 \mathrm{kpa}$; the mean type-1 intrabubble pressure was $10.16+/-3.65 \mathrm{kpa}$. The mean bursting pressure of a type-1 BB was $66.65+/-18.65 \mathrm{kpa}$, while that of a type-2 BB was $14.77+/-2.44 \mathrm{kpa}$. The volume of air required to create a type-1 BB was $0.54 \mathrm{ml}$ and the volume of a type-1 $B B$ was consistently $0.1 \mathrm{ml}$.

Conclusions Dua's layer baring DALK can withstand high intraoperative pressures compared to Descemet's membrane baring DALK. The study suggests that it could be safe to undertake procedures such as DALKtriple with a type-1 BB but not with a type-2 BB.

Eye (2018) 32, 146-151; doi:10.1038/eye.2017.121; published online 30 June 2017
Introduction

Deep anterior lamellar keratoplasty (DALK) has replaced penetrating keratoplasty as the procedure of choice in surgical management of eyes with diseases affecting the corneal stroma and affecting sight such as scars, dystrophy or ectasia. The big bubble (BB) technique ${ }^{1}$ is the most popular technique wherein air is injected in the corneal stroma to separate either the Descemet's membrane (DM) or the DM together with a layer of deep corneal stroma termed the pre-Descemet's layer (Dua's layer-DL). This allows excision of the affected stroma and recipient epithelium and replacement with healthy stroma and epithelium from a cadaver donor.

When air is injected in the corneal stroma, it either cleaves the DL from the deep stroma to create a big bubble termed type- 1 or it accesses the plane between DM and DL to create a thin walled bubble termed type-2. The wall of a type- 1 BB is made of DL and DM, while that of a type-2 $\mathrm{BB}$ is made of $\mathrm{DM}$ alone and is more vulnerable to major tears or bursting during surgery. Often during injection of air, tiny bubbles escape from the peripheral cornea, in the vicinity of the trabecular meshwork, into the anterior chamber and can cause post-operative raised intraocular pressure. ${ }^{2-4}$

Dua $e t a l^{5}$ have reported that DL is a strong and resilient layer with bursting pressure 1.45 bars. On the basis of the above information, Zaki AA et $a l^{6}$ described a combination of DALK with phacoemulsification and lens implant, termed the DALK-Triple procedure. When confronted with patients requiring DALK, who also had dense cataracts, they were able to perform cataract surgery under the exposed DL of a type$1 \mathrm{BB}$. They reported that DL could withstand all 
pressure fluctuations associated with the phacoemulsification procedure, and that, despite stromal scarring requiring keratoplasty, the DL was remarkably clear in most cases. ${ }^{6}$ In one instance, they attempted DALK-Triple under the DM (type-2 BB), which burst promptly during injection of viscoelastic in the anterior chamber.

In this study, we report the pressure and volume of air required to create the $\mathrm{BB}$, the volume and pressure of air in the type- $1 \mathrm{BB}$ and the bursting pressure of the type$1 \mathrm{BB}$.

\section{Materials and methods}

\section{Tissue samples}

Twenty-two human sclera-corneal discs from eye bank donor eyes that were not suitable for transplantation were used. The sclera-corneal discs were maintained in organ culture in Eagle's minimum essential medium with $2 \%$ foetal bovine serum for 4-8 weeks post-mortem. Donor details are given in Table 1.

\section{Experiment to measure pressure}

Air injection The sclera-corneal disc was placed endothelial side up in a petri dish and kept moist with balanced salt solution. In fifteen samples, under an operating microscope, a 30 gauge needle, bent to an angle of $135^{\circ}$, bevel up, attached to a $20 \mathrm{ml}$ syringe was passed from the scleral rim into the corneal stroma and advanced to the centre of the disc. The needle was passed close to the endothelial surface without perforating it. Air was injected with force to overcome the tissue resistance, until a big bubble was formed. The type of the bubble was determined, type- 1 or type- 2 . The position of the needle tip was kept constant in the centre of the sclera-corneal disc in mid stroma.

Pressure measurement An electronic pressure gauge/ converter device was used (Keller, K-114, Winterthur, Switzerland). The tube from the device was linked to the side arm of a three-way cannula attached between the syringe and needle. The injecting needle was attached to the front end of the cannula and a $20 \mathrm{ml}$ syringe to the other end. The device was also connected to a personal computer (PC) via a USB port. The USB link also powered the device. Pressure readings were recorded in real time and transmitted as serial RS485 half-duplex signals to the PC, where the pressure was displayed as a continuous trace on the screen by the software associated with the K-114 device. (Figure 1) The pressure recorded was that in the syringe during injection of air. In validation experiments, when the needle was not inserted in tissue and the piston was advanced rapidly, the pressure recorded was between 0 and 1, indicating that the resistance offered by the needle was not relevant to the pressures measured (data not shown).

The maximum pressure required to create the bubble was recorded. The plunger was then released and allowed to attain a stable position. The needle tip was advanced to lie inside the BB and the bubble inflated till its wall was

Table 1 Donor details for the sclera-corneal discs used in the experiments

\begin{tabular}{|c|c|c|c|c|c|c|}
\hline Sample no. & Eye bank no. & Type of big bubble (BB) & Sex & Age & Date of death & Cause of death \\
\hline E1955 & M20599B & T1BB & $\mathrm{F}$ & 67 & $08 / 05 / 2014$ & Stroke \\
\hline E2168 & M21433B & T1BB & $\mathrm{F}$ & 60 & $29 / 12 / 2014$ & Other (unknown) \\
\hline E2182 & M21468A & T1BB & $\mathrm{F}$ & 58 & $07 / 01 / 2015$ & Cancer \\
\hline E2183 & M21468B & T1BB & $\mathrm{F}$ & 58 & $07 / 01 / 2015$ & Cancer \\
\hline E2246 & M21715A & T1BB & $\mathrm{F}$ & 69 & $15 / 03 / 2015$ & Chronic obstructive pulmonary disease \\
\hline E2187 & M21447B & T1BB & $\mathrm{F}$ & 65 & $02 / 01 / 2015$ & Pending \\
\hline E2385 & M22280B & T1BB & M & 73 & $29 / 06 / 2015$ & Respiratory failure \\
\hline E2347 & M22237A & T1BB & $\mathrm{F}$ & 52 & $17 / 06 / 2015$ & Encephalopathy \\
\hline E2278 & M22072B & T1BB & $\mathrm{F}$ & 80 & $07 / 05 / 2015$ & Sepsis \\
\hline E2276 & M22016B & T1BB & M & 74 & $01 / 05 / 2015$ & Brain damage hypoxia \\
\hline E2275 & M22016A & T1BB & M & 74 & $01 / 05 / 2015$ & Brain damage hypoxia \\
\hline E2309 & M21828B & T1BB & M & 72 & $02 / 04 / 2015$ & Cronic obstructive pulmonary disease \\
\hline E2326 & M22034A & T2BB & $\mathrm{F}$ & 75 & $04 / 05 / 2015$ & Myocardial infarction \\
\hline E2348 & M22237B & T2BB & $\mathrm{F}$ & 52 & $17 / 06 / 2015$ & Encephalopathy \\
\hline E2677 & M22933B & T1BB & $\mathrm{F}$ & 81 & $29 / 12 / 2015$ & Myocardial infarction \\
\hline E2675 & M22956B & T1BB & M & 53 & $02 / 01 / 2016$ & Unknown \\
\hline E2674 & M22956A & T1BB & M & 53 & $02 / 01 / 2016$ & Unknown \\
\hline E2678 & M22913A & T1BB & $\mathrm{F}$ & 44 & $14 / 12 / 2015$ & Intracranial heamorrhage \\
\hline E2679 & M22913B & T1BB & $\mathrm{F}$ & 44 & $14 / 12 / 2015$ & Intracranial heamorrhage \\
\hline E2829 & M23226B & T1BB & M & 80 & $14 / 03 / 2016$ & Cancer \\
\hline E2836 & M23301B & T1BB & $\mathrm{F}$ & 88 & $03 / 04 / 2016$ & Old age \\
\hline
\end{tabular}


a

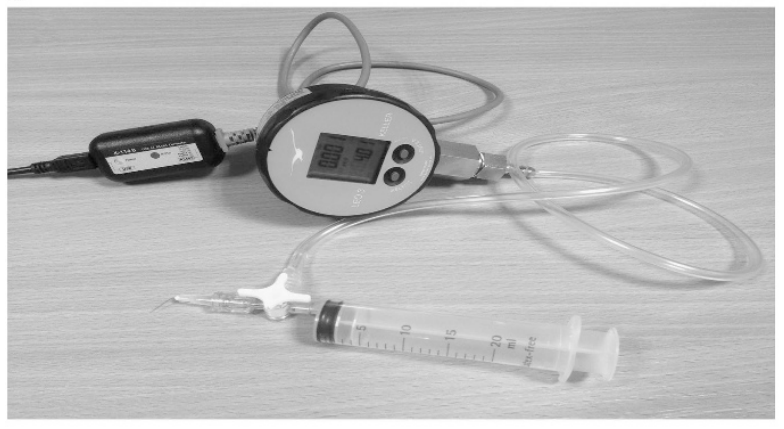

b

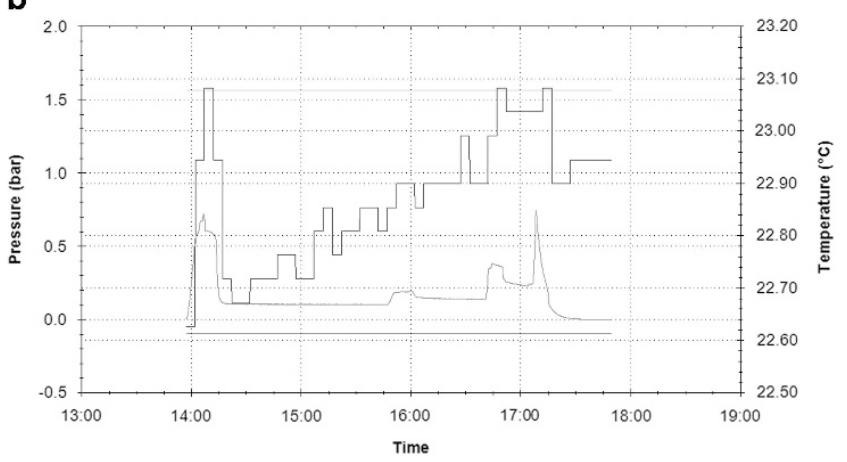

Figure 1 (a) Pressure converter system K-144, (b) Real pressure record over time (red graph), the temperature of the pressure sensor (blue graph), the maximum pressure (pink graph), the minus pressure (green line). A full colour version of this figure is available at the Eye journal online.
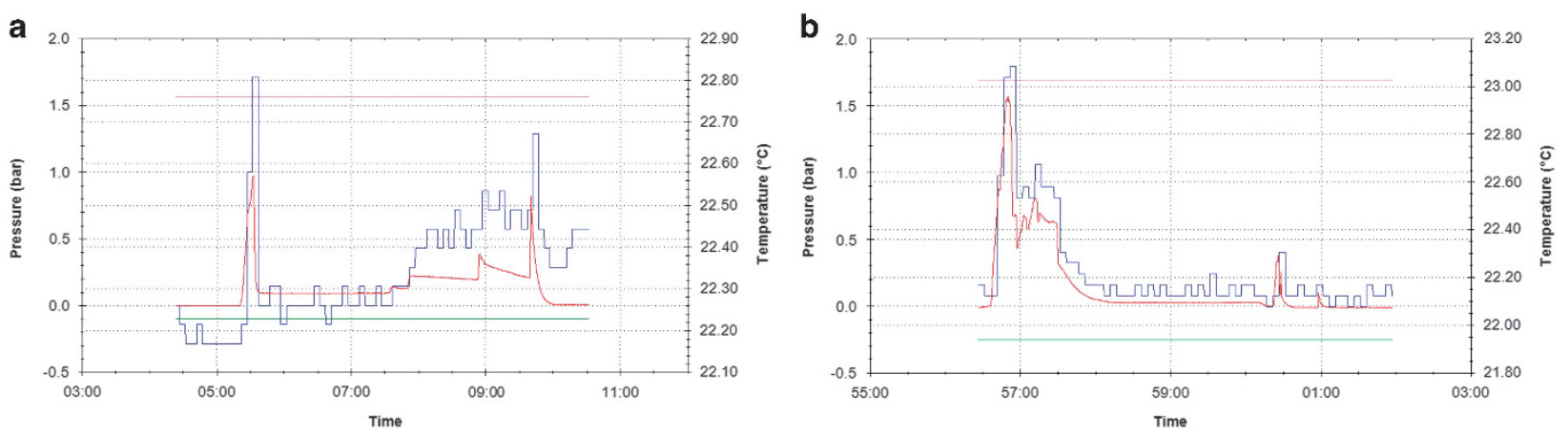

Figure 2 (a) Pressure change over time (red line) in T1BB. (b) Pressure change over time (red line) in T2BB.

taut. The pressure recorded at this point was taken as the base line intrabubble pressure. With the needle tip in the $\mathrm{BB}$, the piston was pushed further with force until the BB burst. This recorded the bursting pressure of the bubble (Figures 2a and b).

\section{Experiment to measure volume}

As air leaked through multiple points along the circumference of the corneal periphery, a clamp was designed to block the holes and stop air leak. In seven samples, the sclera-corneal discs were clamped in a circular clamp of $10 \mathrm{~mm}$ diameter that prevented air escape from the periphery. A 30 gauge needle attached to a $1 \mathrm{ml}$ syringe (internal diameter $5 \mathrm{~mm}$ ) filled with air was passed into the corneal stroma from the scleral rim as described above. During injection, the maximum compression of air (position of piston) at the time air just started to appear in the corneal stroma was recorded. The piston was held in place until a type-1 BB was formed. The pressure on the piston was released and piston was allowed to reverse to a stable position. The volume of air lost in the cornea was ascertained from the final position of the piston. The BB diameter was measured with a pair of surgical callipers. The needle was then advanced into the BB and all the air aspirated until the BB had completely collapsed. This provided a measure of the volume of air in the big bubble. The pressure (above atmosphere) in the syringe at the point where air started to emerge in the tissue from the needle tip was deduced by the formula P1V1=P2V2, where P1 is the initial pressure (atmospheric) and V1 the initial volume $(1 \mathrm{ml})$, and P2 is the final pressure (unknown) and V2 the final volume (mean $0.54 \mathrm{ml}$, see results).

\section{Results}

The average age of donors was 66 years (range; $52-80$ years). There were 15 females and 7 males.

\section{Pressure measurements}

Twelve type- 1 and 3 type-2 BB were obtained (Table 2). The mean pressure attained to create a BB was $96.25+/$ - $21.61 \mathrm{kpa}$ (range 90-130 kpa). For type-1 BB, the mean intrabubble pressure was $10.16+/-3.65 \mathrm{kpa}$ (range 5.2-18 kpa) and the bursting pressure was 66.65 +/ - 18.65kpa (range 40-110kpa). The median bursting pressure was $68.5 \mathrm{kpa}$ (Table 2). Accurate measurements of type- 2 BB could not be obtained as when advancing the 
Table 2 Measurements of the big bubble

\begin{tabular}{cccc}
\hline Sample no. & $\begin{array}{c}\text { Diameter } \\
(\mathrm{mm})\end{array}$ & $\begin{array}{c}\text { Intrabubble pressure } \\
(\mathrm{Kpa})\end{array}$ & $\begin{array}{c}\text { Bursting } \\
\text { pressure }(\mathrm{kpa})\end{array}$ \\
\hline T1BB & & & \\
E1955 & $\mathrm{nm}$ & $\mathrm{nm}$ & 45 \\
E2168 & 7 & 12 & 60 \\
E2182 & 9 & 13 & 80 \\
E2183 & 8.5 & 14 & 73 \\
E2246 & 8.5 & 11.6 & 66 \\
E2187 & 8.5 & 18 & 40 \\
E2309 & 8.5 & 7.5 & 110 \\
E2275 & 8.5 & 7.5 & 78 \\
E2276 & 8.5 & 7.5 & 55 \\
E2278 & $\mathrm{nm}$ & 5.2 & 71 \\
E2347 & 8.5 & 6.8 & 76.8 \\
E2385 & 8.5 & 8.7 & 45 \\
& & & \\
T2BB & & & 17 \\
E2326 & 10 & $n m$ & 12 \\
E2348 & 10.5 & $n m$ & \\
E2384 & 10.5 & $n m$ & \\
\hline
\end{tabular}

Abbreviations: Kpa, kilopascal; nm, not measured.

needle into the bubble cavity, while the needle was still in the stroma, the type-2 BB burst in one case and the DM disinserted (separated along its peripheral attachment to the stroma) in one sector before the bubble could be inflated enough to make the DM taut. The mean pressure at the time of the type-2 BB burst/disinserted was 14.77 +/ - 2.44 kpa (range 12.0-17.0 kpa) (Table 2).

\section{Volume measurements}

In the bubble volume experiment, the maximum compression of air required to create type- $1 \mathrm{BB}$ was 0.54 $+/-0.07 \mathrm{ml}$ (range $0.5-0.7 \mathrm{ml}$ ), the volume of air lost in the cornea was $0.38+/-0.06 \mathrm{ml}$ (range $0.3-0.5 \mathrm{ml}$ ) and the average volume of the $\mathrm{BB}$ was $0.1 \mathrm{ml}$. The mean pressure in the syringe at which air started to emerge in the tissue, as calculated from the volume compression, was $131.82+$ / - $50.58 \mathrm{kpa}$ (range 101.28-236.3 kpa above atmosphere). The difference in pressures measured directly with the gauge and by this method were not statistically significant $(P=0.25)$ (Figure 3$)$.

\section{Statistical methods}

The data was normally distributed as confirmed by Levene's test. Statistical analysis between two groups was performed by the unpaired student $t$-test using Graphpad prism version 5.0. (Graphpad software, La Jolla, CA, USA). $P<0.05$ was considered statistically significant.

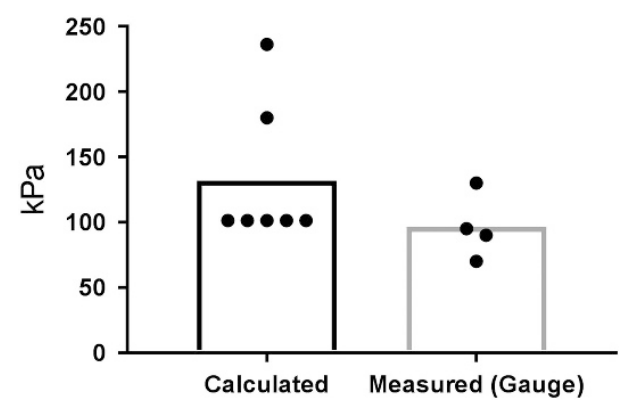

Figure 3 Compares the pressure calculated from the volume compression of the syringe and that measured directly with gauge $(P=0.25)$.

\section{Discussion}

In DALK by the BB technique, when air is injected in the corneal stroma, a type-1 BB forms by air cleaving in the plane of deep stroma and DL, with a posterior displacement of DL and DM. The cleavage and displacement are related to the pressure of air in the corneal stroma and in the BB. As the BB expands posteriorly, the intrabubble pressure is countered by the intraocular pressure, which can rise up to $70 \mathrm{~mm}$ of mercury (authors' unpublished observations). This counter pressure and the closed space within which the $\mathrm{BB}$ expands limits the posterior expansion of the BB in the eye thus rupture of a type- $1 \mathrm{BB}$ during inflation is unlikely and has not been reported. However, when the type-1 BB is deflated and the corneal stroma anterior to it is removed, the DL+DM bulge anteriorly to assume a convex dome shape. Any pressure applied to the DL+DM from within the eye, as during the DALK-triple procedure, would cause the layers to expand outward, into the atmosphere and theoretically reach a bursting point. In this study, we set out to ascertain the minimum and mean popping (bursting) pressure of the layers to establish whether it would always be safe to perform cataract surgery under DL+DM after creating a type-1 BB.

The pressure converter K-114 allowed us to measure in real time the pressure at the tip of the needle during the creation of a BB. On initiation of injection, air is compressed in the syringe on account of the tissue resistance offered by the corneal stroma at the site of the tip of the needle. Once this is overcome, air starts to enter the stroma separating the lamellae, and the intrastromal pressure builds up as the cornea gets completely aerated. At a critical tissue pressure, the air forces its way to the plane anterior to DL and cleaves this away from deep stroma as a type- $1 \mathrm{BB}$. The volume of air required to achieve the critical tissue pressure depends on the escape of air through the trabecular meshwork or through distinct peripheral holes in the stroma, during injection. ${ }^{7-9}$ This confounder was eliminated by the use of the clamp, 
which prevented any escape of air, thus giving us an accurate measure of the mean tissue pressure required to create a BB overcoming tissue resistance, which was 96.25 $+/-21.61 \mathrm{kpa}$. It has been recently demonstrated that air injected in the corneal stroma follows a consistent path regardless of the location, direction of bevel, and depth of the needle tip in the stroma. ${ }^{9}$

Once a type- 1 BB was created, the intrabubble pressure was ascertained by advancing the needle into the cavity of the BB. This measured $10.16+/-3.65 \mathrm{kpa}$. In the ex vivo situation of this study, it was possible to expand the type$1 \mathrm{BB}$ to its bursting point by continued forceful injection of air with the needle positioned in the cavity of the bubble. This situation would simulate increased intraocular pressure exerted on the layers during phacoemulsification carried out under the layers (DALKtriple). The lowest pressure at which a type- 1 BB burst was $40 \mathrm{kpa}$ and the highest was $110 \mathrm{kpa}$. The mean bursting pressure was $66.65+/-18.65 \mathrm{kpa}$. Although we reported the bursting pressure in our original paper, ${ }^{5}$ we refined the measurement by placing the needle tip in the type- $1 \mathrm{BB}$ while increasing the pressure to the bursting point. This approach eliminated any variations induced by the resistance of the stroma to the passage of air. Any effect of variable leakage of air from the periphery of the sclera-corneal disc was prevented by the use of the clamp. In addition, the accuracy of the measurements was enhanced by using the continuous digital pressure recording device.

A number of studies have reported the variations in intraocular pressure during phacoemulsification. By direct measurements during surgery, Zhao $\mathrm{Y}$ et al ${ }^{10}$ found that the IOP fluctuated from 13-96 mm Hg (1.8$13.5 \mathrm{kpa})$. Khng $\mathrm{C}$ et al stated that IOP exceeded $60 \mathrm{~mm} \mathrm{Hg}(8.4 \mathrm{kpa})$ and the highest IOP occurred during hydro-dissection, viscoelastic injection and intraocular lens insertion. ${ }^{11}$ Vasavada $\mathrm{V}$ et al compared the impact of different fluidic parameters on intraoperative IOP and found that the minimum IOP in the low and high parameters groups was $35 \mathrm{~mm} \mathrm{Hg}(4.9 \mathrm{kpa})$ and $34.5 \mathrm{~mm} \mathrm{Hg}$ (4.8 kpa), respectively, and the maximum IOP in the low and high parameters groups was 69 (9.7 kpa) and 85 (11.9 kpa) $\mathrm{mm} \mathrm{Hg}$ respectively. ${ }^{12}$ In another study, Kamae KK et al ${ }^{13}$ monitored IOP during IOL implantation and found that the mean and peak IOPs exceeded $60 \mathrm{~mm} \mathrm{Hg}$ (8.4 kpa) during IOL implantation. In comparison, the data on bursting pressure of the DL+DM generated in this study show that the pressures attained during cataract surgery are several times less than what is required to burst the layers under which phacoemulsification can be carried out in the DALK-triple procedure. Even the lowest bursting pressure had a safety margin of over $25 \mathrm{kpa}$ (177.5 mm Hg) compared to the highest pressure reached during phacoemulsification. This would indicate that DALK-triple is a viable option with regard to the risk of inadvertent rupture of the DL+DM layers intraoperatively.

When cataract and DALK surgery are required simultaneously, if the cornea is clear, one could consider performing phacoemulsificaton as the first step and DALK as the second step of the same procedure. However, when the cornea is scarred to an extent that visualisation is poor, a triple-DALK would be the preferred option. With triple-DALK, when air injection fails to produce a type- $1 \mathrm{BB}$, manual dissection allows access to the plane between the deep stroma and DL. Once the opaque cornea, related to the aeration of the stroma anterior to the DL, is removed, the transparent DL allows phacoemusification to be carried out.

We were able to create both type- 1 and type- $2 \mathrm{BB}$, as reported by Dua et al; however, the type- $1 \mathrm{BB}$ was more consistent, occurring in $86.4 \%$ of the 22 sclera-corneal discs. The data provided in this study can help us develop an automated system whereby we can produce big bubbles in vivo with improved consistency.

\section{Summary}

What was known before

- Dua's layer is a tough, resilient and elastic layer, which can be bared during DALK by the pneumo-dissection big bubble technique.

- Reports showed that DALK-triple procedure can be performed safely with phacoemulsification by baring Dua's layer due to its resiliency and strength.

What this study adds

- The maximum pressure required to burst Dua's layer is less than that reached during phacoemulsification surgery, which makes it possible to perform DALK-triple safely.

- Descemet's membrane bursts easily at a very low pressure. This makes it risky to attempt the DALK-triple with type-2 $\mathrm{BB}$ because the intraocular pressure during phacoemulsification is higher than what can be withsood by the Descemet's membrane.

\section{Conflict of interest}

The authors declare no conflict of interest.

\section{Acknowledgements}

This work was funded by The Royal College of Surgeons, Edinburgh, Scotland, Royal Blind Scotland and The Elizabeth C King Trust, Pittsburgh, USA. 


\section{References}

1 Anwar M, Teichmann KD. Big-bubble technique to bare Descemet's membrane in anterior lamellar keratoplasty. J Cataract Refract Surg 2002; 28: 398-403.

2 Tan DT, Dart JK, Holland EJ, Kinoshita S. Corneal transplantation. Lancet 2012; 379: 1749-1761.

3 Espandar L, Carlson AN. Lamellar keratoplasty: a literature review. J Ophthalmol 2013; 2013: 894319.

4 Maurino V, Allan BD, Stevens JD, Tuft SJ. Fixed dilated pupil (Urrets-Zavalia syndrome) after air/gas injection after deep lamellar keratoplasty for keratoconus. Am J Ophthalmol 2002; 133: 266-268.

5 Dua HS, Faraj LA, Said DG, Gray T, Lowe J. Human corneal anatomy redefined: a novel pre-Descemet's layer (Dua's layer). Ophthalmology 2013; 120: 1778-1785.

6 Zaki AA, Elalfy MS, Said DG, Dua HS. Deep anterior lamellar keratoplasty-triple procedure: a useful clinical application of the pre-Descemet's layer (Dua's layer). Eye 2015; 29: 323-326.

7 Dua HS, Faraj LA, Said DG Dua's layer: its discovery, characteristics and clinical applications In: Ángeles del Buey, Sayas MA, Cristina Peris Martinez CP (eds). Biomechanica y Arquitetura Corneal. Elsevier: Barcelona 2014, pp 35-47.

8 Dua HS, Faraj LA, Said DG. Dua's layer: discovery, characteristics, clinical applications, controversy and potential relevance to glaucoma. Expert Rev Ophthalmol 2015; 10: 531-547.

9 Dua HS, Faraj LA, Kenawy MB. Dynamics of big bubble formation in deep anterior lamellar keratoplasty (DALK) by the big bubble technique: In vitro studies. Acta Ophthalmol 2017; e-pub ahead of print 8 May 2017; doi:10.1111/ aos.13460.

10 Zhao Y, Li X, Tao A, Wang J, Lu F. Intraocular pressure and calculated diastolic ocular perfusion pressure during three simulated steps of phacoemulsification in vivo. Invest Ophthalmol Vis Sci 2009; 50: 2927-2931.

11 Khng C, Packer M, Fine IH, Hoffman RS, Moreira FB. Intraocular pressure during phacoemulsification. J Cataract Refract Surg 2006; 32: 301-308.

12 Vasavada V, Raj SM, Praveen MR, Vasavada AR, Henderson BA, Asnani PK. Real-time dynamic intraocular pressure fluctuations during microcoaxial phacoemulsification using different aspiration flow rates and their impact on early postoperative outcomes: a randomized clinical trial. J Refract Surg 2014; 30: 534-540.

13 Kamae KK, Werner L, Chang W, Johnson JT, Mamalis N. Intraocular pressure changes during injection of microincision and conventional intraocular lenses through incisions smaller than $3.0 \mathrm{~mm}$. J Cataract Refract Surg 2009; 35: 1430-1436. 